must go on ... first the blade, where we are to-day; then the ear, where we shall be to-morrow ; then the full corn in the ear, which awaits our children's children, and which we live to hasten."

\title{
SOME OBSERVATIONS AND REMARKS ON THE AIR-CURRENTS IN NASAL RESPIRATION.
}

\author{
By Charles A. Parker, F.R.C.S. Edin., \\ Surgeon to the Throat Hospital, Golden Square, London.
}

Since the respiratory functions of the nose have been fully investigated and understood, and the peculiar vascular structure of the inferior turbinates has been demonstrated, it has been assumed that the air during nasal inspiration comes into contact with these bodies, the structural peculiarities of which seem especially suited to yield both warmth and moisture. It is said ${ }^{2}$ that the function of the inferior turbinated bodies appears to be that of increasing the area of mucous membrane over which the air passes when either more heat or more moisture is demanded; that after exposure to cold these bodies are found to assume their greatest proportions, whilst in an atmosphere supersaturated with moisture they are frequently found to be collapsed. Again, it is said that in hot dry rooms these bodies swell so as to saturate the air with moisture as it passes over their surface. Such statements as these are quite common, and while not wishing to deny that the inferior turbinates vary in size in response to the varying conditions of the atmosphere, I do not think that the air comes to any great extent into direct contact with these bodies.

There has always seemed to me considerable difficulty in accepting the idea that the inspired air travels through the lower part of the nasal cavities, and on the following grounds :

1. Patients with an absolutely free inferior meatus and postnasal space will often complain of stuffiness and inability to breathe through the nose.

2. Hyperplastic or cedematous enlargement of the middle turbinates, especially of their anterior ends, or muco-purulent catarrh affecting the middle meatus, may cause difficulty of nasal respiration, although the lower passages may be unusually patent.

3. Polypi will cause marked nasal obstruction even in slight cases, where the inferior meatus is quite free.

1 "The Ascent of Man," chap. x., by H. Drummond.

"MacDonald, "The Respiratory Functions of the Nose," London, 1889, p. 51. 
4. A very slight enlargement of the adenoid tissue in the postnasal space will cause considerable difficulty to nasal inspiration. On examining such a case with a post-rhinoscopic mirror, the whole of the inferior meatus and lower turbinates is easily seen, and often the greater part of the middle passages. The adenoids may only hide the upper fourth of the septum, and yet obstruction will be complained of. Even in cases of great enlargement of the adenoid tissue, it is quite rare to find the lower passages hidden from view.

These facts suggest that nasal inspiration takes place, at all events to a very great extent, through the middle meatuses. On looking into the literature of the subject, I am unable to find anything very definite or conclusive as to the course which the air takes on passing through the nose. Some observers have held that it traversed the lower meatus, whilst others have thought that it passed through the middle passage.

Paulsen $^{1}$ divided the head of a cadaver in the middle line, and distributed small pieces of red litmus-paper about the nasal passages, and having replaced the halves of the head, he drew air saturated with ammonia through the nose by means of a pump fixed in the trachea, and noted in what regions the litmus-paper was turned blue. G. Franke, ${ }^{2}$ having divided a head in the middle line and removed the septum, replaced the latter by a plate of glass, and then by means of a pump placed in the trachea drew smoke through the nose, and watched its course through the glass. In both these experiments the air was found to traverse chiefly the middle meatus. Franke concludes that the air on entering the nose sweeps upwards towards the roof, over the middle and superior turbinates, and then curves downwards towards the roof of the naso-pharynx, the air in the inferior meatus meanwhile remaining practically stationary. During expiration he considers the air takes much the same course in the reverse direction, except that the upward curve is not so great.

MacDonald ${ }^{3}$ assumes, from the disposition, shape, and anatomy of the nostrils, as well as from the pathological conditions found in the pharynx in cases of enlarged middle turbinates, that the course of the inspiratory current is through the middle meatus. Paulsen and Franke performed their experiments on the cadaver, which render them a little unsatisfactory, whilst MacDonald,

1 Sitzungsb. d. k. Akad. d. Wissensch., Wien, 1882 (3), Bd. LXXXV., S. 348.

2 Archiv. f. Laryngol. u. Rhinol., Berlin, 1893, Bd. I., s. 236.

3 "Nasal Obstruction," London, 1887, p. 13. 
though he advances a clever and interesting chain of argument, brings forward no fact to clinch it.

It has therefore seemed to me worth while to make some investigations in order, if possible, to determine the natural air-way during nasal respiration in the living subject, and to see if any useful points can be deduced therefrom.

The method I have adopted for inspiration is to ask my subjects to sit in front of me and to breathe absolutely naturally through the nose, whilst I, on my part, have held a Kabierskie's insufflator filled with lycopodium (the lightest innocuous powder I could think of) about 6 inches from their nostrils, and I have gently puffed the lycopodium into the air they breathed. After examining the result of this, I have asked them to breathe deeply through the nose whilst I puffed the lycopodium more liberally. As regards expiration, I have made my subjects smoke cigarettes and gently exhale the smoke through the nose, whilst I watched its course by means. of a nasal speculum.

Applying these methods, I have examined normal noses and cases of spurs, deflections, hypertrophic rhinitis, rhinitis sicca, ozæna, and adenoids, and I think the examination has disclosed a few points of interest.

The following is a summary of my observations:

1. In Normal Nares. - During natural quiet inspiration the powder, carried by the air, impinges first on the septum at the "site of predilection" for nasal hæmorrhage and for simple perforations-i.e., a little less than half an inch within the nostrils and about one third of an inch from the floor of the nose. It then shoots upwards and slightly backwards, and spreading itself in a broad band over the septum in the middle fossa, impinges on the middle turbinated body, especially at its anterior end. The outer wall of the middle passage also often receives some of the powder, and when a good view can be obtained, the powder will be seen stretching up into the superior meatus as far as the eye can reach. The powder entirely misses the inferior meatus and lower turbinated body. Occasionally a little may be seen on the upper surface of the latter, but it must be remembered that, though light, lycopodium is heavier than air, and it has appeared to me that what settles in this position has been carried there by its own weight. It may therefore be accepted, I think, as a fact that in all cases of normal nares the inferior meatus will be free from powder, and even in abnormal noses I have not seen any deposited in the channel formed by the under surface of the inferior turbinate above, and the floor of the nose below. On examining the post- 
nasal space with a mirror, if powder has penetrated as far back, it will be seen quite at the top of the posterior choanæ, and possibly on the upper fourth of the septum, but no lower. Some will also be seen on the roof of the naso-pharynx.

After deep and forcible inspiration, the distribution of the powder is much the same as on quiet inspiration, only in addition some may be seen at the upper part of the internal surface of the lower turbinated and more on its upper surface. None, however, is ever seen on its under surface or on the floor of the nose. Posteriorly, considerable quantities will be seen on the roof of the naso-pharynx and some on the Eustachian cushions, as well as on the upper part of the septum. If inspiration has been sufficiently forcible, some powder will be seen in the centre of the posterior wall of the pharynx, and in a few cases, where enough has passed through to show well, it has seemed to follow the $V$-shaped distribution of the lymphoid tissue on the posterior wall, so often seen in children with adenoids, coming to an apex at the centre of the oral portion of the pharynx. From this point the powder takes a straight course into the larynx, impinging on the arytenoids and processus vocales, missing, as a rule, the epiglottis.

The course of the expired air is a little more difficult to determine accurately, but, as far as can be gathered by the smoke-test, it seems that expiration takes place, at all events to a very great extent, through the inferior meatus, if the inferior meatus be defined as that portion of the nasal cavities below the upper level of the inferior turbinated body.

If the subject of experiment exhale the smoke very slowly, it apparently comes out along the floor below the under surface of the lower turbinate; if exhalation is moderately forcible, it comes through the whole of the inferior meatus, as defined above; if it is quite forcible, it seems to come through both middle and inferior passages, but always more abundantly, more rapidly, and in a more compact stream through the latter. I think it is evident that whilst inspiration takes place through the middle and superior passages, the expired air passes chiefly through the inferior passage. It will be noticed that this conclusion is somewhat at variance with that arrived at by Franke, who thinks that expiration also takes place through the middle fossa, only on a lower level than inspiration.

2. In Cases of Spurs.-If a spur is situated fairly low down, and does not protrude very far forward, and even though it is in firm contact with the inferior turbinate, it does not make any difference whatever to the distribution of the powder during in- 
spiration. During expiration the smoke-stream is generally split into two, one half passing above the spur and the other half below the spur.

If the spur, on the other hand, is situated higher up, or if it is prolonged forward to or beyond the 'site of predilection,' then the distribution of the powder is considerably altered. The anterior end of the spur is thickly coated, and the stream is diverted on to the inferior turbinate, the anterior end or inner and upper surfaces getting powdered according to the position and size of the outgrowth. In spite of the obstruction some of the powder senerally finds its way into the middle meatus, especially during forcible inspiration, in which case its distribution is as in a normal nose, and if any powder finds its way into the post-nasal space it will be seen on the roof. Expiration may be partly above and partly below, or entirely below, the spur, depending apparently on its position.

3. In C'ases of Ineriation of the Septum. - The exact curve, extent, and position of the deviation in each particular case influences markedly the distribution of the powder. It will collect on any deviation which prevents the free entrance of air into the middle meatus. After striking the deviation, the powder may pass on to the upper surface of the inferior turbinate or on to the outer wall of the middle meatus; but a good deal of it generally passes upwards and strikes some portion of the under surface of the middle turbinated body.

As regards expiration in cases of deviation, no rule can be laid down, but it is most frequently through the inferior meatus.

4. In Cas's of Turgescence and IIypertrophies of the Inferior. Turbinated Bodly.-The inferior turbinated body may be very considerably enlarged without in any way affecting the distribution of the powder. It shoots straight above it, and becomes deposited in the middle and upper fosse as in a normal nose. If the anterior end is markedly enlarged so as to obstruct the air-way into the middle meatus, it will then become covered with powder, and the amount in the middle meatus will be diminished or absent according to the size and position of the enlargement. In some cases the stream of powder will be turned into the inferior meatus between the lower turbinate and septum, but not between it and the floor, and in other cases the bulk of the powder will pass along the lower part of the middle passage, but little penetrating to the higher regions. Moderate enlargement of the posterior end of the inferior turbinate does not apparently affect inspiration.

On expiration, the behaviour of the smoke varies according to 
the exact condition of the inferior turbinate. If the condition is such as to obstruct the inferior meatus, the smoke dribbles from above the inferior turbinate in a diffuse manner, instead of in a straight stream. This occurs whether the passage is blocked in its whole length or whether the obstruction is at either its anterior or posterior end. Enlargement of the posterior end of the inferior turbinate has a marked effect on the smoke, destroying the stream and diverting it into the middle meatus. Artificial stenosis of the anterior portion of the inferior meatus, produced by plugs of cottonwool, has the same effect.

5. In Hypertrophies of the Middle Turbinated Body.-If the lower passage is clear, a large amount of powder will be seen on the enlarged middle turbinate on inspiration, and none can be seen beyond it, unless the passages are very roomy. In the post-nasal space the powder, when it does find its way through, has a rather lower distribution than in normal cases. The smoke in expiration is unaltered.

6. In Rhinitis Sicca.- - In these cases, as would be expected, the anterior portion of the septum and the anterior end of the middle turbinated body are especially covered with powder.

7. In Cases of Ozcena the powder is deposited as in a normal nose.

8. In Cases of Polypi. - When polypi obstruct the middle meatus, the powder impinges against the anterior end of the inferior turbinate, and also becomes entangled amongst the polypi.

9. In Adenoid Grouths. - In cases of adenoids much depends upon their size. In slight cases, the anterior nares being normal, the powder passes through the middle meatus as usual, and impinges on the anterior surface of the growth, and, collecting there, can be seen with a mirror. In more aggravated cases a great deal of powder passes along the inferior meatus, showing chiefly on the lower part of the septum and inner surface of the inferior turbinated body. Little, if any, reaches the post-nasal space, even on forcible inspiration, but becomes entangled in the growths. Expiration is not appreciably interfered with in slight cases, and even in marked cases not nearly so much so as inspiration.

\section{ReMarks.}

Various interesting points seem to me to arise out of the above observations, which may be discussed under the same headings.

1. Normal Noses. - From the distribution of the powder in ordinary quiet nasal inspiration, we may conclude that inspiration 
takes place through the middle and superior passages of the nose, and that very little air comes into direct contact with the inferior turbinated body. This suggests, firstly, that the inferior turbinated body keeps the residuary air (that is, the air remaining stationary in the nose between the end of expiration and the commencement of inspiration) saturated with moisture and warmed to blood-heat, and that the inspiratory current is rarmed from this rather than by being directly exposed to the inferior turbinated body itself; and, secondly, that possibly the mucous membrane covering the septum and middle and superior turbinates plays a more important part in warming the inspired air than is usually supposed, and that hence its conservation in operative surgery is most probably important.

As regards expiration, judging from the smoke-stream, it may be concluded that it occurs through the inferior meatus chiefly. Seeing that in no case, with either normal or abnormal nares was any powder found in these experiments to be deposited below the inferior turbinated body, it may be assumed that this region of the nose plays no part whatever in inspiration. Is it possible that the function of the inferior meatus is connected with the efficient drainage of the nasal cavities? The secretions from all parts of the nose naturally tend to gravitate towards the inferior meatus, and the constant flow of air during expiration would tend to force the secretions towards the nostrils. This would not be counteracted by the inspiratory stream, as it takes a different course. Added to this, if on blowing the nose the expiratory blast comes chiefly through the inferior meatus, where the discharges have collected, it has a narrow channel to traverse, and so can gain sufficient ris a tergo and swiftness to force before it the collected secretions, thus resembling shooting a pea from a pea-shooter. If, on the other hand, the expiratory blast were distributed over all the nasal chambers, it would be like trying to shoot a pea from the barrel of a gun.

Again, as regards olfaction, it seems probable that odorous substances are carried sufficiently high by the ordinary air-stream to stimulate the special nerve-endings. Recent investigations have shown that the true olfactory mucous membrane is limited to a small area on the upper part of the superior turbinated body, with a corresponding area on the septum (A. von Brunn ${ }^{1}$ ). It has been assumed from Paulsen's and Franke's experiments that this area is above the air-way. Haycroft ${ }^{2}$, in his article on the Sense of Smell

${ }^{1}$ Arch. f. mikr. Anat., Bonn, 1892, Bd. XXXIX., S. 632.

2 "Text-book of Physiology," edited by Schïfer, Edinburgh and London, 1900 , vol. ii., pp. 1248-9. 
in Schïfer's "Physiology," explains the stimulation of this senseorgan by the gradual displacement of the residual air in the upper part of the nasal cavities by air coming from the main air-current, which latter, if laden with odorous substances, would thus reach and stimulate the sense-organ. Now, if a powder as heavy as lycopodium is carried in perfectly ordinary nasal inspiration well up into the superior meatus, and probably higher, surely odorous substances, which, it may be assumed from their behaviour in air, are always lighter than air, would be carried well up into the highest meatus, and so come into contact with the special nerve-endings; and the short forcible sniffs given when one desires to distinguish the odour of any particular thing would undoubtedly bathe the sensitive region with the odorous substances.

Lastly, the course of the air-way in the pharynx and larynx, as shown by the deposition of the powder, also suggests points of interest. The fact that in the pharynx the air seems to follow the distribution of the adenoid tissue is suggestive that the function of the latter structure is, as is often surmised, to deal with the impurities of the air, especially those of a bacterial nature. The fact that the air during inspiration impinges on the arytenoid bodies, and not straight on the vocal cords, may have a purpose in protecting the latter structures from the direct irritation of the air and foreign particles.

2. Spurs and Ieriations.-As far as nasal inspiration is concerned, the above observations seem to point to the necessity of operative interference only when spurs and deviations are so situated as to obstruct the direct route of the air into the middle meatus. As far as I can judge, the majority of spurs are situated sufficiently low down and far back as to be out of the way of the air-stream, and therefore, as far as breathing is concerned, they may be left alone. Deviations, on the other hand, are a far more frequent source of obstruction, even very slight deviations, which one is in the habit of looking upon as hardly worth interfering with. Dislocation of the anterior triangular cartilage is also productive of very great obstruction, often preventing any powder at all entering the nostril, and therefore urgently requiring surgical interference. I think these facts are corroborated by clinical observations. Spurs are constantly discovered in patients utterly unaware of any nasal trouble. These will be found to be situated low down and not very far forward. Simple deviations, or deviations with spurs, are found to be productive of considerable trouble, as are also dislocations of the anterior triangular cartilage. The inspiration of lycopodium may be put to a practical use, for it 
lodges chiefly on those parts which are causing obstruction to the normal air-way, and one is therefore enabled to see exactly those parts which should be removed or reduced in size in order to remove the nasal stenosis. I have already found this most useful, for in this way it has been possible to limit the operation to those parts actually causing the obstruction, and thus to preserve much useful mucous membrane.

As regards expiration, it may occasionally happen that a spur, though so situated as not to interfere with inspiration, may render expiration uncomfortable and even difficult, in which case operation will be necessary.

The above remarks apply to spurs and deviations in relation to the air-way. Operative interference is also said to be necessary on account of their mechanical irritation causing chronic rhinitis, and, secondarily, chronic otitis, pharyngitis and laryngitis. Of this I am very doubtful. Clinically, I fancy, one never sees spurs producing these secondary manifestations unless the air-way is interfered with. As already mentioned, spurs not causing difficulty of breathing are very constantly found in people not complaining of any trouble whatever.

3. Inferior T'urbinated Bodies.-My observations on these cases quite confirm what I believe has now become fully recognised, namely, that in order to relieve nasal stenosis it is sufticient to remove either the anterior end of the inferior turbinate or the posterior end, or both, but that it is never necessary to remove the whole body. It must be remembered that the anterior and posterior ends of the lower fossa of the nose are anatomically narrow, whereas the middle portion is comparatively wide, so that, even if the middle part of the inferior turbinate is enlarged, it produces less ill result. For the relief of difficult inspiration, the anterior end most frequently requires attention, and for the relief of difficult expiration the posterior end may have to be dealt with. Great turgescence or hypertrophic outgrowths of the inferior turbinate may also cause difficulty of expiration, in which case they will require treatment. We have seen that in a normal nose practically no powder is deposited on the anterior end. Should it therefore be found to be thickly coated with powder at the expense of the middle meatus, it may be taken for granted that it is a source of obstruction, and it should consequently be dealt with.

4. Enlargements of the Middle Turbinated Bodies.-As far as nasal inspiration is concerned, my observations lead me to believe that enlargements of the middle turbinates are as deleterious as, if not more so than, a similar condition of the inferior turbinates. 
Patients are often met with who complain of stuffiness of the nose to such an extent as to necessitate buccal breathing, and yet who can, on being told to do so, shut the mouth and breathe comparatively easily through the nostrils. In such, the middle turbinates will be found to be enlarged, and if lycopodium is used it will be seen that their anterior ends and under surfaces are thickly coated with powder. When these conditions are found, it is advisable to remove the anterior ends of the middle turbinates, so as to restore the air-way into the upper fossæ, which are normally used in quiet nasal inspiration.

5. Polypi. - The normal air-way being chiefly through the middle meatus, it is easy to see why even small polypi should produce considerable distress in breathing. They should of course be removed for this as well as for other reasons.

6. Rhinitis Sicca.-In this disease the two favourite places for the formation of crusts are the "site of predilection " on the septum and on the anterior end of the middle turbinates. These are the two places on which the lycopodium powder most readily and chiefly collects during inspiration, and therefore it may be concluded that they are the places on which dust and other foreign particles carried by the air would most readily impinge, causing irritation, dryness and crusts.

7. Laryngitis Sicca.-In this trouble the crusts are most commonly seen covering the arytenoid bodies, the interarytenoid space, and the vocal processes. Here, again, it is just the parts on which the lycopodium powder impinges during ordinary inspiration which become dry and crusted. In this may be traced cause and effect.

8. Ozcena.-MacDonald ${ }^{1}$ suggests that an up-turning of the nostrils diverts the air-stream along the inferior meatus, and hence the up-turning of the nose to avoid an unpleasant smell. This suggested to me that possibly in the typical ozænic patient, in whom the nose is anatomically upturned, the air might take a lower course than normal, and that this conceivably in some measure might account for the drying of the secretions. Observations with lycopodium in cases of ozæna, however, show that the air-stream takes the usual course.

9. Adenoids.-The normal course of the air-stream apparently sweeps over the vault of the naso-pharynx. This fact, and the collection of powder between the posterior choanæ and the pad of growth, explain why a small overgrowth of adenoid tissue considerably interferes with the normal air-way, producing nasal stenosis. It is interesting to note that in bad cases of adenoids 1 “Nasal Obstruction," pp. 13, 14. 
the air-stream takes a lower course, as shown by powder being deposited on the inner and upper surfaces of the inferior turbinated body.

\section{Sumiary.}

These observations show :

1. That during quiet inspiration in a normal nose the air traverses the middle, superior, and probably the fourth meatus.

2. That inspiration is impeded by:

(a) Spurs and deviations of the septum and enlargements of the inferior turbinated body, if they project forward and upwards. (For practical purposes I think a rule may be laid down that if such abnormalities cross and break an imaginary line drawn from the anterior extremity of the inferior meatusi.e., just internal to the vestibule-to the anterior end of the middle turbinate, they will cause obstruction.)

(b) Enlargements of the middle turbinated body, polypi, etc.

(c) Hypertrophies and growths springing from the vault of the naso-pharynx.

3. That in expiration the air traverses chiefly the inferior meatus.

4. That expiration will be more especially affected by:

(a) Hypertrophies of the posterior end of the inferior turbinate.

(b) Hypertrophies, etc., causing stenosis of the inferior meatus.

\section{PROCEEDINGS OF THE BRITISH LARYNGOLOGICAL, RHINOLOGICAL, AND OTOLOGICAL ASSOCIATION.}

General Meeting, May 10, 1901.

Mr. Mayo Collier, President, in the Chair.

Mr. Lennox Browne showed a patient, who had been exhibited at the last meeting, suffering from a lympho-sarcoma growing from the back wall and rather to the right of the pharynx. This had now been removed, a tracheotomy having been previously performed and a Hahn's cannula introduced. The tumour, which 